\title{
Marble Market: Bimanual Interactive Game with a Body Shape Sensor
}

\author{
Kentaro Fukuchi ${ }^{1}$ and Jun Rekimoto ${ }^{2,3}$ \\ ${ }^{1}$ Graduate School of Information Systems \\ The University of Electro-Communications \\ Choufu-shi, Tokyo , JAPAN 182-8585 \\ fukuchi@megaui.net \\ ${ }^{2}$ Interfaculty Initiative in Information Studies, \\ The University of Tokyo \\ ${ }^{3}$ Interaction Laboratory \\ Sony Computer Science Laboratories, Inc. \\ rekimoto@acm.org
}

\begin{abstract}
A video game application was developed using SmartSkin, a body shape sensing device. The video game uses a table-sized SmartSkin that can recognize players' arms on the tabletop. Sensor values are translated to a virtual potential field and the system calculates dynamics of game characters on the field.

At most four players can play the game, and the players control many independent game characters displayed on the table using their arms simultaneously.
\end{abstract}

\section{Introduction}

We developed a multi-player video game that allows users to control many independent game characters simultaneously with player's arms. In order to realize bimanual manipulation on a table-sized input surface, we employed a body shape sensing device based on capacitive sensing, and we introduced a new bimanual interaction technique which we called "bulldozer manipulation". This technique uses an interpolated potential field created by sensor inputs, and calculates physical dynamics of characters on the potential field.

In our game application, the bulldozer manipulation allows users to move more than 10 game characters simultaneously. Players can gather, guide or block game characters with their arms intuitively.

\section{System Architecture}

\subsection{Sensing Device}

We employed SmartSkin[5], a human-body sensing device based on capacitive sensing. This sensor recognizes multiple hand positions and shapes and calculates the distance betwenn the hand and the surface.

SmartSkin consists of grid-shaped electrodes, transmitters, receivers and micro controllers. Figure 3 shows the principle of operation of SmartSkin. The vertical wires are 


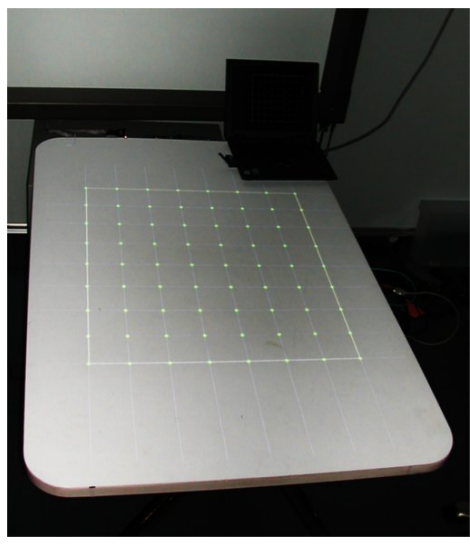

Fig. 1. Table-sized SmartSkin

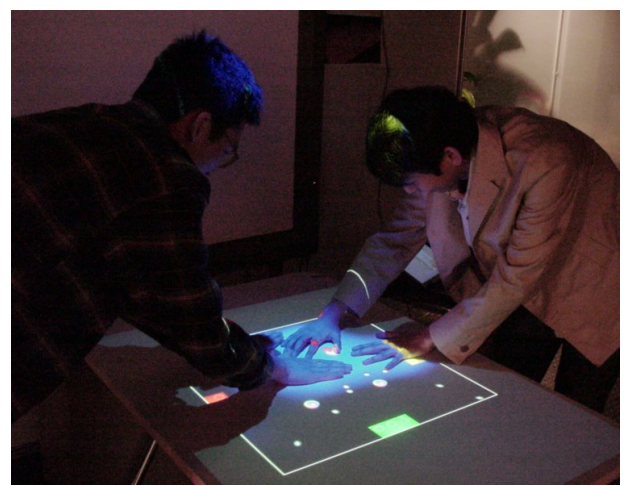

Fig. 2. Two players playing Marble Market

transmitter electrodes, and the horizontal wires are receiver electrodes. When one of the transmitters is excited by a wave signal (of typically several hundred kilohertz), the receiver receives this wave signal because each crossing point (transmitter/receiver pairs) acts as a (very weak) capacitor. The magnitude of the received signal is proportional to the frequency and voltage of the transmitted signal, as well as to the capacitance between the two electrodes. When a conductive and grounded object approaches a crossing point, it capacitively couples with the electrodes, and drains the wave signal. As a result, the received signal amplitude becomes weak. By measuring this effect, it is possible to detect the proximity of a conductive object, such as a human hand.

The time-dividing transmitting signal is sent to each of the vertical electrodes, and the system independently measures values from each of the receiver electrodes. These values are integrated to form two-dimensional sensor values. The right of Figure 5 shows the the integrated sensor values.

The integrated sensor values is similar to a two-dimensional image. The distance between the human body and the sensor corresponds to the luminance of a pixel. Once these values are obtained, algorithms for image processing can be applied to them to recognize gestures.

We used a table-sized SmartSkin that has $8 \times 9$ grid cells on a table top, and each cell is $10 \times 10 \mathrm{~cm}$. The resolution of the sensor data is 10 bits $(0-1,023)$ and the scan rate is 10 scans per second. A piece of thin white plywood was placed on the grid, and a computer screen was overlaid on it using a projector set above the table (shown in Figure 1). This system provides a touch-panel-like input surface, but can recognize multiple hands of users.

\subsection{Interaction Techniques}

We developed an interaction technique which is called "bulldozer manipulation" with SmartSkin and introduced to our game application.

Fitzmaurice et al. reported that when they provided a pile of colored LEGO blocks and asked subjects to separate them by color, the subjects slid the blocks simultaneously 


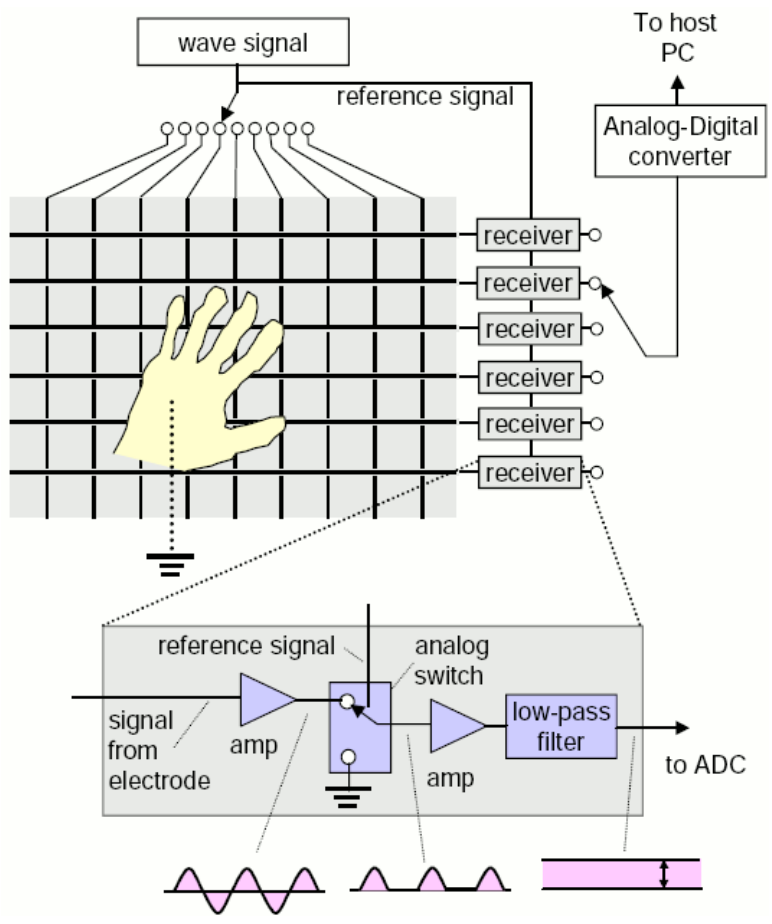

Fig. 3. SmartSkin sensor configuration

rather than of picking up and dropping them, using their hands and arms like a 'bulldozer' [2]. Bulldozer manipulation can be performed by physical contact between the objects and the user's hand, and this kind of manipulation was not possible on the input system without physical devices.

In order to simulate the bulldozer manipulation on SmartSkin, the fact that SmartSkin can recognize a shape of the user's arm on the input surface is considered. By capturing the arm shape the system can track the posture of the hands on the input surface so that it can calculate collisions between virtual components and the hands. However, it is difficult to track a hand from a shape data. There are many known techniques to estimate the posture of a hand from video images, but it is not possible to apply these techniques to shape data for the following reasons. First, the posture of the user's arm changes dynamically, but since the shape data from SmartSkin is too simple it is difficult to estimate the motion between the frames, while a video image provides many features such as a texture of the skin. Second, the shape data from SmartSkin represents very limited posture information, and even distinguishing the left and right hand from the data is difficult when the hand only partially touches the input surface. Third, the multi players may touch the input surface simultaneously, but SmartSkin can not distinguish them.

For these reasons, rather than estimating the posture of the hands, bulldozer manipulation was implemented by creating a potential field from the shape data. 


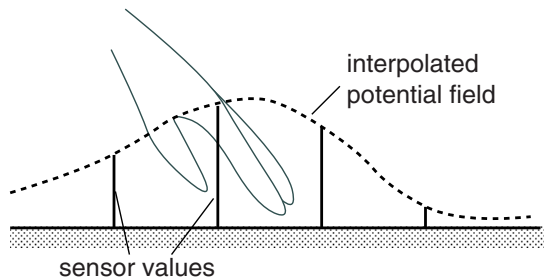

variation of sensor value-hight field mapping

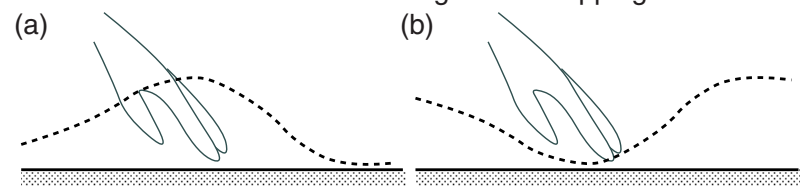

Fig. 4. Two methods of creating a potential field
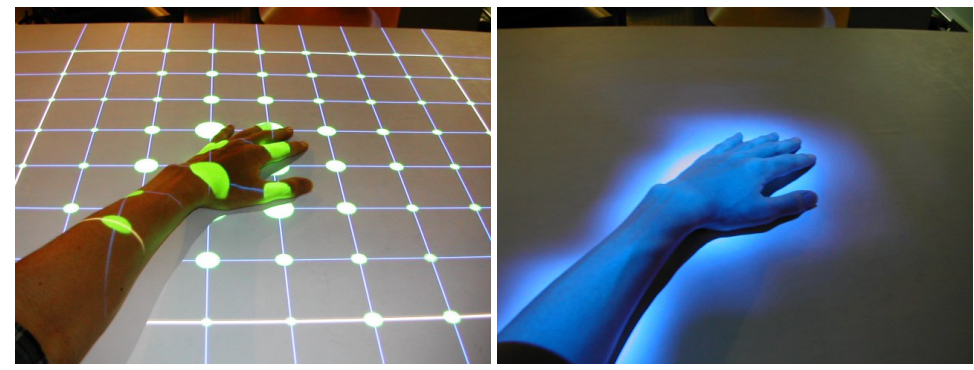

Fig. 5. Interpolated sensor values

When a user touches the input surface with his hands, the shapes of the hands are recognized as $8 \times 9$ sensor values. The shape data is smoothed using a bicubic convolution interpolation (Figure 5). This smoothed surface was used as a potential field and the dynamics of objects on the potential field were calculated so that the objects move on the field according to the position and shape of the hands simultaneously. Each object, which is treated as a point mass, rolls on the potential field toward the lower position.

There are two method by which to create a potential field from the sensor values. Figure 4 shows these methods. Method (a) makes the potential high when a hand is close to the surface, which means that an object on the potential field moves away from the hand. In contrast, method (b) makes the potential low when a hand is close to the surface, which means that an object is drawn to the hand. Both methods were tested for bulldozer manipulation, and method (a) was found to be better for this purpose.

\section{Video Game Application}

\subsection{Game Rules}

The game screen is shown in Figure 6 The goal of this game is to bring as many small balls, called Marbles, as possible to the player's Basket. A Marble Machine produces 


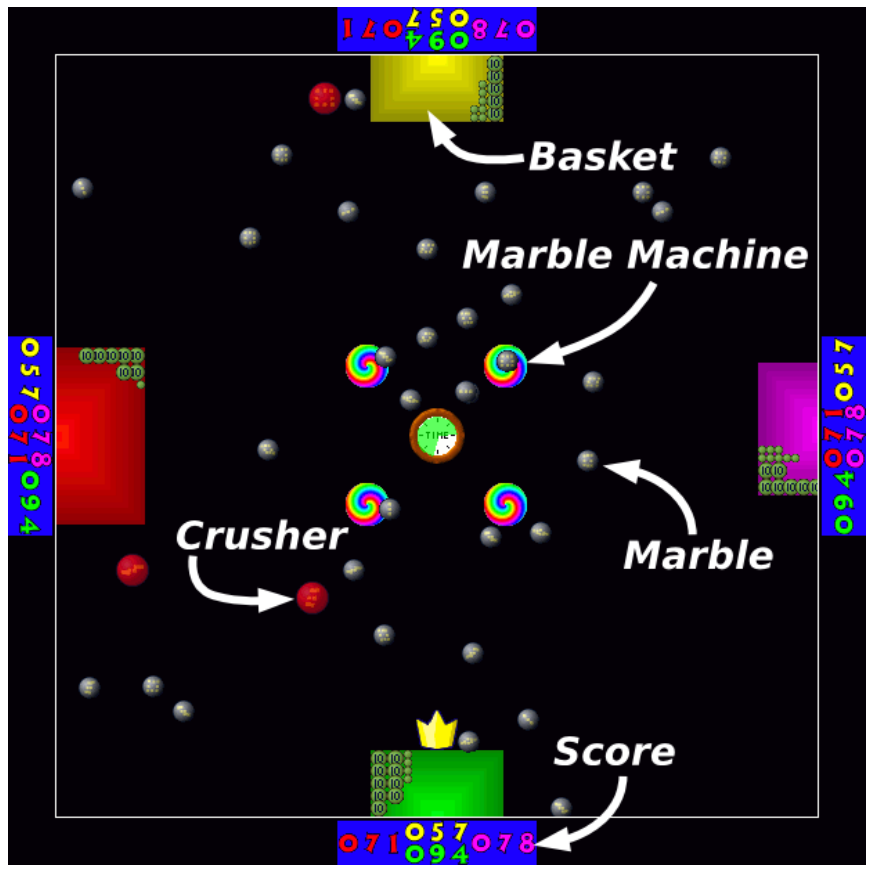

Fig. 6. An illustration of the game field

marbles continuously. The Marble Machine sometimes produces large red balls called Crushers. Crushers break Marbles. When into a Basket, the Basket looses a quarter of the Marbles already collected. Therefore, players should not collect Crushers, but rather send them toward his opponents' Basket. Marble Machines sometimes provide Gold Marbles, which have the value of 50 Marbles, or Special Marbles, which bring about some special event.

The player who collected the most Marbles in two minutes wins the game.

\subsection{Concurrent Manipulation of Many Marbles}

Marble Market was exhibited at Sony ExploraScience in Tokyo. In addition, Marble Market was exhibited in a number of open laboratory events, and several visitors played this video game. At both exhibitions, the basic rules of the game were described and the method of moving the Marbles by hand were instructed. However, a detailed description of the techniques of concurrent manipulation was not given. Despite this, the players discovered various techniques by themselves. Through these exhibitions, a number of typical techniques of concurrent manipulation of Marbles were observed.

In order to gather Marbles, the player basically places their of both hands on the table and moves slowly toward his Basket. Many players were observed to place their hands beside a group of Marbles and make them roll on the potential field towards their Basket, following the Marbles with their hands to adjust their direction. Some players formed a funnel in front of their Basket so as to guide Marbles into their basket. 


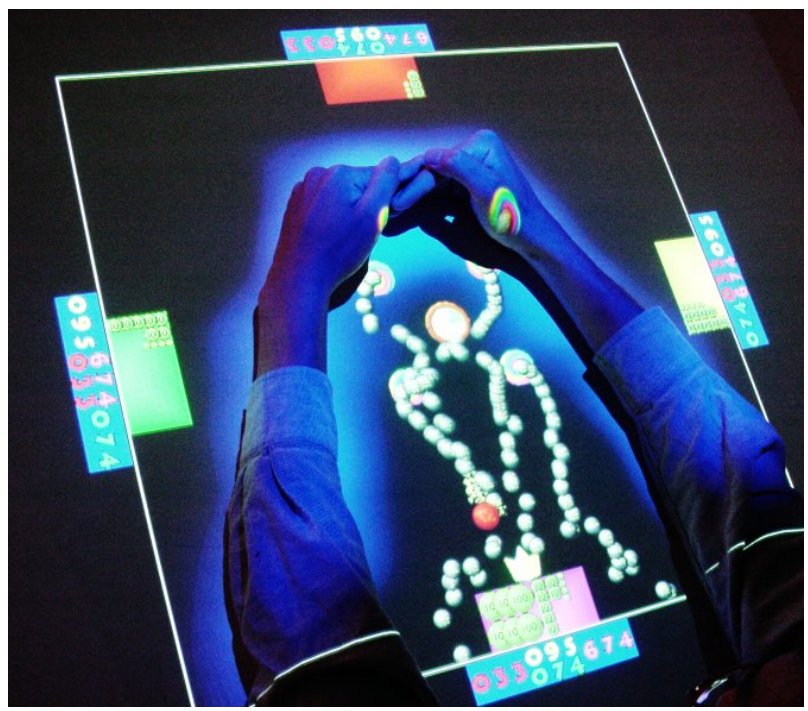

Fig. 7. Gathering marbles by arms

The SmartSkin detected not only hands but also arms. When players discovered this, they gathered Marbles more aggressively. Several of them moved their entire arms in the manner of a bulldozer blade. Some players used both arms. Moreover, some players closed off the Marble Machines and Baskets using their arms, as shown in Figure 7 In this way, all of the Marbles from the Marble Machines were guided into their Basket.

These manipulation techniques can be used when a potential increases under a hand. In contrast, when the potential decreases under a hand, as shown in (b) of Figure 4 the following techniques are effective. One technique is simply placing a hand over a Basket to form a potential hole over it, so as to pull in the Marbles rolling near the Basket. The other is putting a hand on a Marble Machine for a while in order to collect the Marbles in the hand, and then to bring them to a Basket by moving the hand slowly.

As mentioned above, techniques by which to gather Marbles differed between the two different potential field. These methods must be evaluated in order to discuss their advantages, but the former method was employed for this application from the observation of the user tests. The former method enables intuitive manipulation, and allows various manipulation, that avoids monotony and makes the game more exciting.

\section{Discussion}

From the observation in the exhibitions, the bulldozer manipulation of Marble Market is easy to learn and effective for concurrent manipulation of many Marbles. As an example of ease of learning, early elementary school children soon learned to control the Marbles using their arms.

On the other hand, in some cases, the behavior of the Marbles was not so intuitive for the players. First, most players moved their hands from the Marble Machines to the Baskets too quickly at first. When the hand is moved quickly, it overtakes the Marbles 
and the Marbles are accelerated to the opposite side, as described in Section 2.2 In the current implementation, the hand should be moved at the same speed as the Marbles, or placed as shown in Figure 7. This indicates that the potential field method limits bulldozer manipulation. Second, some players pushed the table top surface strongly to block Crushers from entering their Baskets. Since SmartSkin does not sense pressure, this motion is meaningless. However, this observation suggests the use of a pressure sensor for a more intuitive game interface.

\section{Related Work}

Matsushita et al. developed HoloWall[4], an interaction system with an infra-red camera. The system consists of a rear-projection screen and an infra-red camera that is placed behind the screen. The user's arms or hands on the screen is captured by a infraled camera behind the screen. Two-handed interaction was introduced on Holowall, and a shape-based input is implemented, but it is based on a simple collision detection.

DiamondTouch[1] is a multi-user interaction system that accepts concurrent twohanded input. DiamondTouch can identify who is touching the input surface, however it allows a very limited bimanual input.

Jeff Han et al. introduced a multi-touch sensing technique that utilizes an internal reflection in a clear acrylic board[3]. Their system recognizes a contact surface between the acrylic board and the user's fingertips. Since it does not recognize user's body above the board, it is difficult to recognize the whole shape of the arm on the input surface.

\section{Conclusion}

A video game application, Marble Market, was developed that allows bulldozer manipulation to control several characters concurrently. The application uses a SmartSkin sensor to create a potential field from the position and shape data of players' arms and hands, and then the game characters are forced from the field. Marble Market has been exhibited, and various techniques were observed for concurrent manipulation of the characters. From these observations, the bulldozer manipulation is judged to be easy to learn and effective for concurrent manipulation of multiple components.

\section{References}

1. Dietz, P.H., Leigh, D.: DiamondTouch: A Multi-User Touch Technology. In: Proceedings of UIST'01, pp. 219-226 (2001)

2. Fitzmaurice, G.W.: Graspable User Interfaces. PhD thesis, Dept. of Computer Science, University of Toronto (1996)

3. Han, J.Y.: Low-Cost Multi-Touch Sensing Through Frustrated Total Internal Reflection. In: UIST '05: Proceedings of the 18th annual ACM symposium on User interface software and technology, pp. 115-118. ACM Press, New York (2005)

4. Matsushita, N., Rekimoto, J.: HoloWall: Designing a Finger, Hand, Body, and Object Sensitive Wall. In: Proceedings of UIST'97, pp. 209-210 (1997)

5. Rekimoto, J.: SmartSkin: An Infrastructure for Freehand Manipulation on Interactive Surfaces. In: Proceedings of CHI2002, pp. 113-120 (2002) 